\title{
Non-intubated robotic-assisted thoracic surgery for tracheal/airway resection and reconstruction safe: editorial commentary
}

\author{
Donatas Zalepugas $^{1}$, Philipp Schnorr ${ }^{2}$, Joachim Schmidt ${ }^{1,2}$, Benedetta Bedetti $^{2}$ \\ ${ }^{1}$ Division of Thoracic Surgery, Department of General, Thoracic and Vascular Surgery, Bonn University Hospital, Bonn, Germany; \\ ${ }^{2}$ Department of Thoracic Surgery, Helios Hospital Bonn/Rhein Sieg, Bonn, Germany \\ Correspondence to: Benedetta Bedetti. Department of Thoracic Surgery, Helios Hospital Bonn/Rhein Sieg, 53123 Bonn, Germany. \\ Email: benedetta.bedetti@gmail.com. \\ Comment on: Li S, Ai Q, Liang H, et al. Non-intubated Robotic-Assisted Thoracic Surgery for Tracheal/Airway Resection and Reconstruction: \\ Technique Description and Preliminary Results. Ann Surg 2021. [Epub ahead of print]. doi: 10.1097/SLA.0000000000004887.
}

Submitted Sep 26, 2021. Accepted for publication Oct 13, 2021.

doi: $10.21037 / \mathrm{atm}-21-5128$

View this article at: https://dx.doi.org/10.21037/atm-21-5128

In the article entitled "Non-intubated Robotic-Assisted Thoracic Surgery for Tracheal/Airway Resection and Reconstruction: Technique Description and Preliminary Results", published on Annals of Surgery, the authors report their preliminary results of robotic-assisted thoracic surgery (RATS) non-intubated airway resection and reconstruction under spontaneous ventilation. Five advanced surgical procedures of the thoracic trachea, main carina, left secondary carina, and left main bronchus are presented. A 3-port robotic setup was used. The anesthesia was performed without muscle relaxant, under spontaneous ventilation with laryngeal mask assistance and $60 \%$ oxygen flow. The total time between preparation of the robotic system and operative time ranged from 5 h $5 \mathrm{~min}$ to $9 \mathrm{~h} 55 \mathrm{~min}$. The postoperative hospital stay ranged from 4 to 14 days. None of the patients had complications during the first month after the surgery, and postoperative bronchoscopic examination showed good anastomotic healing and no anastomotic stenosis. Therefore, this article aims to show an example of a combined surgical and anesthesiologic minimally invasive approach with fast-track recovery protocols.

In the "early days" of lung surgery, surgeons started by performing wedge resections and even lobectomies on awake patients with a variety of local anesthesia and nerve blocks. This continued until the 1950s when double-lumen endotracheal tubes seemed to offer a better anesthetic option with lung isolation (1).
In the era of enhanced recovery protocols and evolving minimally invasive thoracic surgery, non-intubated video assisted thoracoscopic surgery (NI-VATS) is having a revival again. For smaller procedures NI-VATS already has an acceptance in the thoracic surgery community and even more complex procedures as NI-VATS lobectomies and sleeve resections are being performed in an increasing number of centers with promising results $(2,3)$. Furthermore, the paper's authors have demonstrated that tracheal and airway surgery with a NI-VATS approach is feasible and manageable with good results in the anastomosis healing and procedural time (4).

Recently, RATS is spreading growingly worldwide and is becoming a minimally invasive alternative to conventional VATS. Again, data has been published that suggest even superior outcomes in RATS in lung cancer surgery (5).

Other protocols and outcome data of small cohorts have been already published regarding sleeve resections, airway and tracheal surgery (6).

Both approaches RATS and VATS seem to offer advantages over the conventional approach, but yet a number of questions have to be asked regarding these new surgical techniques.

The authors of the paper state that their approach combines the robotics arm extra maneuverability and the positive effect of $3 \mathrm{D}$-visualisation with the non-intubated surgery described before in their NI-VATS experience. This kind of approach ensures an optimal surgical vision 
and eliminates the interference of the endotracheal tube, therefore enhancing the reconstruction's accuracy.

The other reported advantage is that mechanically induced ventilation with intubated anesthesia during airway resection and reconstruction is associated with pulmonary damage and risk of airway edema and stenosis of the anastomosis (7). Hence, preventing tracheal intubation should reduce unstable $\mathrm{SpO}_{2}$ intraoperative, avoids time loss caused by cross field ventilation and possible repositioning of the robotic console and results in a shorter in-operating room time.

Along with the mentioned possible benefits of the described non-intubated approach, the possible disadvantages must be considered. First of all, the main anesthesiologic concerns of non-intubated surgery with open airways and moreover circularly resected airways are hypoxemia, hypercapnia and uncontrolled cough $(8,9)$. Hypoxemia may be adequately addressed with a simple elevation of concentration of inhaled oxygen. However, the use of $60 \%$ concentration and more is not recommended as routine practice in surgical patients (10). Furthermore, the use of electrocautery in the presence of high oxygen levels is potentially dangerous. The other concern is the hypercapnia management. There is no feasible way to prevent $\mathrm{CO}_{2}$ cumulation during the operation when the central airway (main bronchus or trachea) is open. Implementation of jet ventilation solves the hypoxemia problem, but not the hypercapnia (11). There is a need for larger volume studies of non-intubated open airway surgery with the of intraoperative collection of blood gas analysis and description of hypercapnia management techniques.

The main surgical concern of non-intubated surgery is severe bleeding during the procedure $(7,12)$. Even though the procedure performed might be an isolated airway resection, there is still a risk of major vessel perforation due to their proximity. A special concern during non-intubated RATS with spontaneous ventilation without application of muscle relaxants is uncontrolled coughing of the patient. This may lead to unpredictable collision of the intrathoracic instruments with nearby blood vessels. In this situation the robotic-surgeon might not be capable to retract the instruments far and fast enough to avoid unwanted tissue damage, because there is a limit to how far the robotic instruments can be quickly withdrawn.

Acute bleeding in minimally invasive procedures is always a threat, but it is manageable. There is a difference between controlled bleeding and uncontrolled bleeding.
In the event of controlled bleeding, compression can be applied, and the surgeon has enough time to assess the situation and, if needed, to convert to an open approach. In the event of uncontrolled bleeding, often compression cannot be applied due to sudden loss of vision (bloody camera/"red out"), lack of space to maneuver, absence of adequate compression instruments in the thoracic cavity at the time of bleeding, or because the bleeding source cannot be identified within a safe time interval. In the "worse case scenario" during a non-intubated RATS, the operating team could be managing an uncontrolled bleeding and complete obstruction of the main airways by blood. It could be difficult to decide which of the problems should be addressed first.

The combined approach described in the paper seems to offer great benefits in the care for patients needing airway or tracheal resection and reconstruction. As well as other novelties in surgery and medicine, these new approaches need to be compared to the conventional surgical technique. Currently, VATS is increasingly used as standard of care in surgery for lung malignancies. The evolution of VATS could either be NI-VATS or RATS. A combination of these strategies should therefore lead to define novel ultra-minimally invasive treatment options (13). The by Li et al. published technique and preliminary results seem to be promising, but in our opinion the role of intubation has to be further examined in studies with greater patient volumes and more complex analysis. A clear management protocol of potentially disastrous intraoperative events needs to be developed before starting with ultra-minimally invasive approaches, but overall the described approach for maximally challenging surgical procedures could be a generally accepted alternative to conventional techniques.

\section{Acknowledgments}

Funding: None.

\section{Footnote}

Provenance and Peer Review: This article was commissioned by the editorial office, Annals of Translational Medicine. The article did not undergo external peer review.

Conflicts of Interest: All authors have completed the ICMJE uniform disclosure form (available at https://dx.doi. org/10.21037/atm-21-5128). The authors have no conflicts 
of interest to declare.

Ethical Statement: The authors are accountable for all aspects of the work in ensuring that questions related to the accuracy or integrity of any part of the work are appropriately investigated and resolved.

Open Access Statement: This is an Open Access article distributed in accordance with the Creative Commons Attribution-NonCommercial-NoDerivs 4.0 International License (CC BY-NC-ND 4.0), which permits the noncommercial replication and distribution of the article with the strict proviso that no changes or edits are made and the original work is properly cited (including links to both the formal publication through the relevant DOI and the license). See: https://creativecommons.org/licenses/by-nc-nd/4.0/.

\section{References}

1. Elkhayat H, Gonzalez-Rivas D. Non-intubated uniportal video-assisted thoracoscopic surgery. J Thorac Dis 2019;11:S220-2.

2. He J, Liu J, Zhu C, et al. Expert consensus on spontaneous ventilation video-assisted thoracoscopic surgery in primary spontaneous pneumothorax (Guangzhou). Ann Transl Med 2019;7:518.

3. Zhang K, Chen HG, Wu WB, et al. Non-intubated videoassisted thoracoscopic surgery vs. intubated video-assisted thoracoscopic surgery for thoracic disease: a systematic review and meta-analysis of 1,684 cases. J Thorac Dis 2019;11:3556-68.

Cite this article as: Zalepugas D, Schnorr P, Schmidt J, Bedetti B. Non-intubated robotic-assisted thoracic surgery for tracheal/airway resection and reconstruction safe: editorial commentary. Ann Transl Med 2021;9(22):1707. doi: 10.21037/ atm-21-5128
4. Jiang L, Liu J, Gonzalez-Rivas D, et al. Thoracoscopic surgery for tracheal and carinal resection and reconstruction under spontaneous ventilation. J Thorac Cardiovasc Surg 2018;155:2746-54.

5. Liang H, Liang W, Zhao L, et al. Robotic Versus Videoassisted Lobectomy/Segmentectomy for Lung Cancer: A Meta-analysis. Ann Surg 2018;268:254-9.

6. Egberts JH, Möller T, Becker T. Robotic-Assisted Sleeve Lobectomy Using the Four-Arm Technique in the DaVinci $\mathrm{Si}{ }^{\circledR}$ and $\mathrm{Xi}{ }^{\circledR}$ Systems. Thorac Cardiovasc Surg 2019;67:603-5.

7. Liang H, Gonzalez-Rivas D, Zhou Y, et al. Nonintubated Anesthesia for Tracheal/Carinal Resection and Reconstruction. Thorac Surg Clin 2020;30:83-90.

8. Huang J, Qiu Y, Chen L, et al. Nonintubated Spontaneous Respiration Anesthesia for Tracheal Glomus Tumor. Ann Thorac Surg 2017;104:e161-3.

9. Okuda K, Moriyama S, Haneda H, et al. Recent advances in video-assisted transthoracic tracheal resection followed by reconstruction under non-intubated anesthesia with spontaneous breathing. J Thorac Dis 2017;9:2891-4.

10. Eskesen TG, Steinmetz J, Rasmussen LS. Using a high inspiratory oxygen fraction during surgery. Ugeskr Laeger 2016;178:V07160474.

11. Akopov A, Kovalev M. Nonintubated Tracheal Surgery. Thorac Surg Clin 2020;30:91-9.

12. Liu L, Mei J, He J, et al. International expert consensus on the management of bleeding during VATS lung surgery. Ann Transl Med 2019;7:712.

13. Pompeo E. Minimally invasive thoracic surgery: new trends in Italy. Ann Transl Med 2015;3:269. 